\title{
Standing waves in fiber-optic interferometers
}

\author{
V. de Haan, ${ }^{1, *}$ R. Santbergen, ${ }^{2}$ M. Tijssen, ${ }^{2}$ and M. Zeman ${ }^{2}$ \\ 'BonPhysics B.V., Laan van Heemstede 38, 3297 AJ Puttershoek, The Netherlands \\ ${ }^{2}$ Delft University of Technology, EWI Faculty, Mekelweg 4, 2628 CD Delft, The Netherlands \\ ${ }^{*}$ Corresponding author: victor@ bonphysics.nl
}

Received 12 May 2011; revised 21 July 2011; accepted 21 July 2011;

posted 29 July 2011 (Doc. ID 147429); published 5 October 2011

\begin{abstract}
A study is presented giving the response of three types of fiber-optic interferometers by which a standing wave through an object is investigated. The three types are a Sagnac, Mach-Zehnder and MichelsonMorley interferometer. The response of the Mach-Zehnder interferometer is similar to the Sagnac interferometer. However, the Sagnac interferometer is much harder to study because of the fact that one input port and output port coincide. Further, the Mach-Zehnder interferometer has the advantage that the output ports are symmetric, reducing the systematic effects. Examples of standing wave light absorption in several simple objects are given. Attention is drawn to the influence of standing waves in fiber-optic interferometers with weak-absorbing layers incorporated. A method is described for how these can be theoretically analyzed and experimentally measured. Further experiments are needed for a thorough comparison between theory and experiment. (C) 2011 Optical Society of America
\end{abstract}

OCIS codes: $\quad 020.1670,060.2310,350.7420$.

\section{Introduction}

The phenomenon of standing waves produced by cotraveling waves in interferometers is a well studied subject (see for instance [1-3]) and also the reflection, transmission, and absorption of traveling waves through objects is well understood (see for instance [4]). However, as soon as two counter traveling waves are considered, the situation is quite different. Wiener [5] and Ives [6] studied light standing waves at a reflecting surface. Silvertooth [7] reports on the construction of a light standing wave sensor. $\mathrm{He}$ inserted a thin light-absorbing layer in a vacuum photo-multiplier tube and detected a varying absorption in the standing wave. Only quite recently Silvertooth's sensor was reproduced by means of transparent thin photodiodes, [요의 enabling the construction of very small interferometers $[10,11]$. The influence of light absorption in standing waves created by interferometers, however, is not comprehensively studied. In this paper, a method is described by which this is made possible. Examples of standing

0003-6935/11/295674-14\$15.00/0

(C) 2011 Optical Society of America wave light absorption in several simple objects are given. Attention is drawn to the influence of standing waves in fiber-optic interferometers with weakabsorbing layers incorporated. A method is described for how these can be theoretically analyzed and experimentally measured.

In general, interferometry is the detection of standing waves in the superposition of two or more wave phenomena. For visible light, the wave phenomena in homogeneous media are mostly regarded as plane waves, which are characterized as function of location $(\vec{r})$ and time $(t)$ by solutions of the Maxwell equations:

$$
\Psi(\vec{r}, t)=\hat{\Psi} e^{i(\phi+\vec{k} \cdot \vec{r}-\omega t)},
$$

where $\omega$ is the frequency of the wave and $\vec{k}$ is the wave vector giving the direction of propagation. The length of the wave vector $k=2 \pi / \lambda$, where $\lambda$ is the wavelength of the light. $\hat{\Psi}$ is the amplitude of the wave and $\phi$ a constant phase. Here, only one component of the electric or magnetic vector field is considered, but the treatment can be extended to include polarization effects. In general, the length of the wave vector and the frequency of the wave are 
coupled via the dispersion relation of the medium in which the wave is traveling:

$$
\omega=k v_{p},
$$

where $v_{p}$ is the phase velocity of the wave. For light, the phase velocity is given by the refractive index of the medium, $n$ and the velocity of light in vacuum, $c$

$$
v_{p}=\frac{c}{n} .
$$

Defined in this way, the refractive index of a medium depends on frequency and can be a complex number. The real part represents the phase velocity and the imaginary part represents the attenuation of the wave traveling inside the medium. The attenuation can be due to absorption or scattering of the wave. For transparent objects like glass or water, the imaginary part is very small and negligible in most cases. For conductive objects like metals, the imaginary part can become much larger than the real part, making those objects strong absorbers.

In the following, waves which can be represented by Eq. (1) are referred to as traveling waves. The intensity of the waves is proportional to the squared modulus of the wave function $|\Psi(\vec{r}, t)|^{2}$. As can be inferred from Eq. (1), for nonabsorbing media, the intensity of a traveling wave is constant. A superposition of two (or more) traveling waves results in interference effects. Let two traveling waves $\Psi_{1}(\vec{r}, t)$ and $\Psi_{2}(\vec{r}, t)$ be superimposed, the resulting wave function is

$$
\Psi(\vec{r}, t)=\hat{\Psi}_{1} e^{i\left(\phi_{1}+\vec{k}_{1} \cdot \vec{r}-\omega_{1} t\right)}+\hat{\Psi}_{2} e^{i\left(\phi_{2}+\vec{k}_{2} \cdot \vec{r}-\omega_{2} t\right)}
$$

The intensity is given by

$$
\begin{aligned}
|\Psi(\vec{r}, t)|^{2}= & \hat{\Psi}_{1}^{2}+\hat{\Psi}_{2}^{2}+2 \hat{\Psi}_{1} \hat{\Psi}_{2} \cos \left(\phi_{1}-\phi_{2}\right. \\
& \left.+\left(\vec{k}_{1}-\vec{k}_{2}\right) \cdot \vec{r}-\left(\omega_{1}-\omega_{2}\right) t\right) .
\end{aligned}
$$

Hence, in general, the intensity is not a constant any more, but depends on both time and space. When the frequency of the traveling waves are taken, the same the time dependence vanishes and only the space dependence remains. In the following this is referred to as a standing wave. Also, when the wave vectors are in the same direction, the space dependence also disappears and the resulting intensity only depends on the phase difference $\phi_{1}-\phi_{2}$. This is the situation encountered in 'standard' interferometers.

In the following, the influence of materials with different dispersion relations on the behavior of standing waves is studied for two situations: a standing wave created by reflection of light to a mirror and a standing wave created by superposition of two counter traveling waves. Interestingly, if the waves travel through a medium with an imaginary part, the behavior of both standing waves is different. The character of this behavior is investigated to get a better understanding of standing light waves to be able to improve fiber-optic interferometer designs.

\section{Reflection at and Transmission through an Object}

The normal reflection geometry to determine the reflection and transmission coefficients is shown in Fig. 1. Here it is assumed that there is no $z$ dependence and that the object is a homogeneous half space with the interface at $y=0$. The incident angle with respect to the interface is $\theta_{i}$ and similar definitions for the reflection and refraction angles $\theta_{r}$ and $\theta_{t}$. To find these angles and the reflection and transmission coefficients, one determines the plane waves in the regions $j(j=0$ for $y>0$ and $j=1$ for $y<0$ ) and then calculates the angles, phases $\phi_{i}$, and amplitudes $\hat{\Psi}_{i}$ of the waves using the assumption that the sum of the plane waves at the interface are continuous and differentiable. This assumption is valid for electromagnetic $s$ waves for any incident angle and for all electromagnetic waves if the incident angle is 0 . The results are the standard Fresnel laws for the reflection and refraction angles and for the reflection and transmission amplitudes [4]. For nonmagnetic media, the Fresnel laws for electromagnetic $p$ waves can be found by replacing the amplitudes of the waves by $\hat{\Psi}_{i} / \sqrt{\epsilon_{i}}$, where $\epsilon_{i}$ is the electric permittivity of medium $i$ [4].

In region 0 exists a standing wave due to the reflection of the incident wave. In region 1 there is no standing wave because the wave is only transmitted. This geometry has to be adapted for the calculation of the amplitudes and phases of the waves in case of incident waves at both sides of the interface. In Fig. 2 the adapted geometry is shown. The homogeneous half space is replaced by a stack of $m$ homogeneous layers made up of different media through which the waves must pass. A wave incident from the bottom of the stack is added. This results in the creation of a standing wave at the bottom side of the object. It is assumed that the medium on the top and on the bottom side of the object is the same and has a refractive index of 1 . Hence, all angles in regions 0 and $m+1$ are the same. In each layer $j$ there is a wave moving from top to bottom and a wave moving from bottom to top. Omitting the $x$ and $t$ dependence, the sum of the waves for $y \geq 0$ is given by

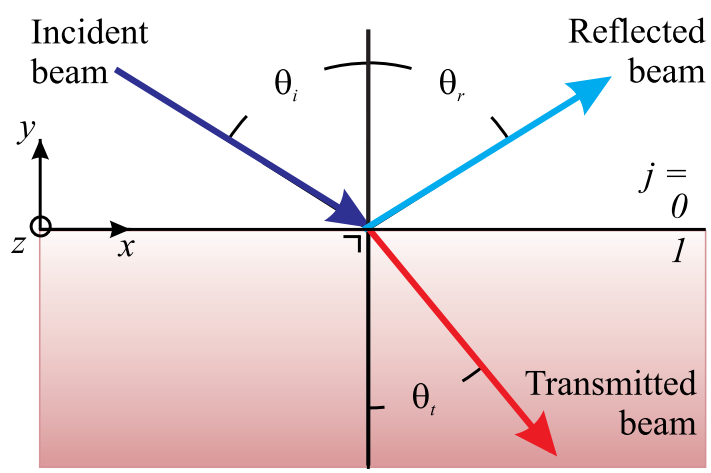

Fig. 1. (Color online) Reflection and transmission of a plane wave with incident angle $\theta_{i}$ on a substrate boundary at $y=0$. 


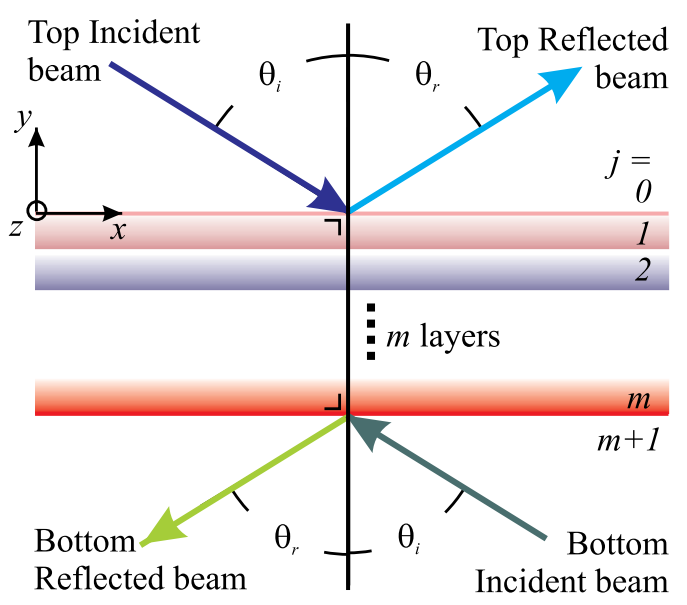

Fig. 2. (Color online) Reflection and transmission geometry for a standing wave at a multilayered object.

$$
\Psi(y)=\Psi_{0}^{+} e^{i q_{0} y}+\Psi_{0}^{-} e^{-i q_{0} y}
$$

and in each layer $j$

$$
y_{j+1} \leq y \leq y_{j}: \Psi(y)=\Psi_{j}^{+} e^{i q_{j}\left(y-y_{j}\right)}+\Psi_{j}^{-} e^{-i q_{j}\left(y-y_{j}\right)},
$$

where $y_{j+1}=y_{j}-d_{j}$ with $y_{1}=0$ and $d_{j}$ equals the thickness of layer $j$. Note that, by this definition the interface between layer $j$ and $j+1$ is at $y=y_{j+1}$. For $y \leq y_{m+1}$,

$$
\Psi(y)=\Psi_{m+1}^{+} e^{i q_{m+1}\left(y-y_{m+1}\right)}+\Psi_{m+1}^{-} e^{-i q_{m+1}\left(y-y_{m+1}\right)},
$$

$\Psi_{j}^{ \pm}$represent the amplitudes and phases of the waves traveling in layer $j$ in the $\pm y$-direction, and $q_{j}$ is the $y$-component of the wave vector of the wave traveling in the $+y$-direction in the medium given by

$$
q_{j}^{2}=k_{0}^{2}\left(n_{j}^{2}-\sin ^{2} \theta_{i}\right),
$$

where $k_{0}$ is the length of the incident wave vector and $n_{j}$ represents the (complex) index of refraction of layer $j$. Note that, for a normal medium (in which no waves are generated) one should take the positive root with a nonnegative imaginary part.

In general, there are four waves which have four different wave vectors. However, when the interfaces are flat and perpendicular to the $y$-axis, there is no momentum change in the $x$-direction, only in the $y$-direction and only the $y$-component of the wave vector has to be considered. Even when the wave vector components parallel to the surface are not equal, interference will occur. In such a case, however, the phase of the waves are not fixed with respect to each other (when the $x$ position is changed) and the interference will be averaged. The averaging depends on the lateral size of the interface and the mismatch between the wave vectors parallel to the surface. For the averaging not having a detrimental effect on the interference amplitude, it is needed that $\theta_{t} \approx 0$.
Note that this is the case if $\theta_{i} \approx 0$. In this case the above equation reduces to

$$
q_{j}=k_{0} n_{j} .
$$

The amplitude and phase of the top incident and reflected beam can be related to the bottom ones by using the continuity and differentiability conditions at each interface. The result is the matrix equation [4].

$$
\left(\begin{array}{c}
\Psi_{m+1}^{+} \\
\Psi_{m+1}^{-}
\end{array}\right)=\left(\begin{array}{ll}
m_{11} & m_{12} \\
m_{21} & m_{22}
\end{array}\right)\left(\begin{array}{c}
\Psi_{0}^{+} \\
\Psi_{0}^{-}
\end{array}\right)
$$

where

$$
\begin{gathered}
\left(\begin{array}{ll}
m_{11} & m_{12} \\
m_{21} & m_{22}
\end{array}\right)=M_{m} M_{m-1} \ldots M_{1} M_{0}, \\
M_{j}=\frac{1}{2}\left(\begin{array}{cc}
1+\frac{q_{j}}{q_{j+1}} & 1-\frac{q_{j}}{q_{j+1}} \\
1-\frac{q_{j}}{q_{j+1}} & 1+\frac{q_{j}}{q_{j+1}}
\end{array}\right)\left(\begin{array}{cc}
e^{-i q_{j} d_{j}} & 0 \\
0 & e^{i q_{j} d_{j}}
\end{array}\right),
\end{gathered}
$$

where $d_{0}=0$. Hence, the matrix (11)determines the coupling of the amplitudes and phases of the top and bottom incident and reflected waves.

Note that, as long as $q_{j} / q_{j+1}$ are real for all $j$, the components of the matrix are correlated as $m_{21}=$ $m_{12}^{*}$ and $m_{22}=m_{11}^{*}$. For the external reflections discussed here, this is the case as long as there are no absorbing materials in the object.

One can rewrite the matrix elements in the form of reflection and transmission amplitudes of the standard geometry. If we set the incident wave at the top to $1\left(\Psi_{0}^{-}=1\right)$ and at the bottom to 0 $\left(\Psi_{m+1}^{+}=0\right)$, then reflection amplitude is given by $\rho_{t}=$ $\Psi_{0}^{+}$and the transmission amplitude by $\tau_{t}=\Psi_{m+1}^{-}$. Hence,

$$
\left(\begin{array}{c}
0 \\
\tau_{t}
\end{array}\right)=\left(\begin{array}{ll}
m_{11} & m_{12} \\
m_{21} & m_{22}
\end{array}\right)\left(\begin{array}{c}
\rho_{t} \\
1
\end{array}\right)
$$

yields $\rho_{t}=-m_{12} / m_{11}$ and $\tau_{t}=1 / m_{11}$, where it was used that the determinant of the matrix is always equal to 1 . The same procedure for the 'standard' reflection at the bottom side

$$
\left(\begin{array}{c}
1 \\
\rho_{b}
\end{array}\right)=\left(\begin{array}{ll}
m_{11} & m_{12} \\
m_{21} & m_{22}
\end{array}\right)\left(\begin{array}{c}
\tau_{b} \\
0
\end{array}\right)
$$

yields $\rho_{b}=m_{21} / m_{11}$ and $\tau_{b}=\tau_{t}=\tau$. Hence, Eq. (11) can be rewritten as

$$
\left(\begin{array}{ll}
m_{11} & m_{12} \\
m_{21} & m_{22}
\end{array}\right)=\frac{1}{\tau}\left(\begin{array}{cc}
1 & -\rho_{t} \\
\rho_{b} & \tau^{2}-\rho_{b} \rho_{t}
\end{array}\right) .
$$

This representation can be used for the design of standing wave experiments. If, for the geometry discussed here no absorbers are used, then $R_{t}=R_{b}=R$ and $T+R=1 \quad$ where $T=|\tau|^{2}, \quad R_{t}=\left|\rho_{t}\right|^{2}$, and $R_{b}=\left|\rho_{b}\right|^{2}$. 


\section{Standing Wave Absorption in an Object}

It is possible that one of the layers of the object discussed in the previous section has a strong imaginary part of the refractive index. An example thereof is a metal layer of a few nanometers thick. Another possibility is a layer in which photons are absorbed and converted into electrons creating a photo current proportional to the absorbed amount of light. The amount of photons absorbed depends on the efficiency of the absorber and the intensity of the electric field. Hence, such an absorbing layer could yield information on the phase of the standing wave. the Poynting vectors of the top and bottom incident waves is used. Then the time-averaged Poynting vector representing the energy density flux incident on the layer from the bottom and top is given by

$$
\langle S\rangle_{\mathrm{ref}}=\frac{c \epsilon_{0}}{2}\left(\left|\Psi_{0}^{-}\right|^{2}+\left|\Psi_{m+1}^{+}\right|^{2}\right)
$$

and the absorbed fraction in layer $j$ can be defined as $\alpha_{j}=A_{j} /\langle S\rangle_{\text {ref }}$. Herein inserting Eqs. (1), (16), (15),

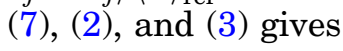

$$
\alpha_{j}=2 k_{0} d_{j} \mathfrak{R}\left(n_{j}\right) \mathfrak{\Im}\left(n_{j}\right) \times \frac{\left|\Psi_{j}^{+}\right|^{2} f\left(-\mathfrak{\Im}\left(q_{j}\right) d_{j}\right)+\left|\Psi_{j}^{-}\right|^{2} f\left(\mathfrak{I}\left(q_{j}\right) d_{j}\right)+2 \mathfrak{R}\left(\left(\Psi_{j}^{+}\right)^{*} \Psi_{j}^{-} f\left(-i \mathfrak{R}\left(q_{j}\right) d_{j}\right)\right)}{\left|\Psi_{0}^{-}\right|^{2}+\left|\Psi_{m+1}^{+}\right|^{2}}
$$

For an electromagnetic plane wave, the dissipation $Q$ is equal to the time-averaged change of the Poynting vector, $\vec{S}$. The wave field of Eq. (1) can be interpreted as the electromagnetic wave. If it is assumed that the absorption is caused by nonmagnetic effects only, then the imaginary part of the permeability will be zero. Photons are absorbed in proportionality to the electric field intensity only. Then, according to equation (80.4) of Landau [12], the absorption (or dissipation) is given by

$$
Q(y)=\omega \epsilon_{0}|\Psi(y)|^{2} \mathfrak{R}(n(y)) \mathfrak{I}(n(y)),
$$

where $\epsilon_{0}$ is the permittivity of free space. $\mathfrak{R}(x)$ is used to denote the real part of $x$ and $\mathfrak{I}(x)$ its imaginary part. $n(y)$ is the refractive index as function of $y$. For each layer $j$ defined in the previous section, $n(y)$ is given by $n_{j}$. To find the absorbed amount in one layer, this equation has to be integrated over the layer thickness

$$
A_{j}=\int_{y_{j+1}}^{y_{j}} Q(y) \mathrm{d} y,
$$

Note that $y_{j}>y_{j+1}$. This absorbed amount must be taken relative to the total available amount, which is given by the time-averaged Poynting vector [12]:

$$
\langle S(y)\rangle=\frac{c \epsilon_{0}}{2}|n(y)||\Psi(y)|^{2} .
$$

From this, it follows that the time-averaged Poynting vector for a standing wave depends on the location where it is determined. In a node, where $\Psi(y)$ is zero, the time-average Poynting vector is also 0 . This means that the energy flux density at this point is 0 . For a single plane wave, the time-averaged Poynting vector is constant. Hence, as a reference to determine the relative amount of absorbed photons, the sum of where

$$
f(x)=\frac{1-e^{-2 x}}{2 x}=1-x+O\left(x^{2}\right),
$$

where $O\left(x^{2}\right)$ is a small quantity of order $x^{2}$. Note that the coefficients $\Psi_{j}^{+}$and $\Psi_{j}^{-}$can be determined by using the appropriate value for $m$ in Eq. (10). If $\left|q_{j}\right| d_{j} \ll 1$, Eq. (19) reduces to

$$
\alpha_{j}=2 k_{0} d_{j} \mathfrak{R}\left(n_{j}\right) \mathfrak{\Im}\left(n_{j}\right) \frac{\left|\Psi_{j}^{+}+\left(\Psi_{j}^{-}\right)^{*}\right|^{2}}{\left|\Psi_{0}^{-}\right|^{2}+\left|\Psi_{m+1}^{+}\right|^{2}} .
$$

This equation shows that, for a first approximation the absorbed fraction in a standing wave is proportional to the thickness of the layer, to the length of the wave vector inside the layer, and the imaginary part of the refractive index. (This is the same for a single traveling wave, which can be inferred from the above equation by taking $\Psi_{m+1}^{+}=0, \Psi_{j}^{+}=0$, and $\left.\left|\Psi_{j}^{-}\right|=\left|\Psi_{0}^{-}\right|.\right)$It is also proportional to $\mid \Psi_{j}^{+}+$ $\left.\left(\Psi_{j}^{-}\right)^{*}\right|^{2}$, which can be reduced to 0 if $\Psi_{j}^{+}=-\left(\Psi_{j}^{-}\right)^{*}$. For larger values of $\mathfrak{\Im}\left(q_{j}\right) d_{j}$, damped interference oscillations can occur due to the last term of Eq. (19). Hence, photon absorption in a standing wave can be similar to or very different from absorption of photons in a traveling wave, depending on both the top and bottom incident waves and the structure and media of the absorbing object.

The dependence on the top and bottom incident waves gives the opportunity to study this phenomena in three geometries. One geometry consists of the object in a Sagnac interferometer. Another one is to put the object in a Mach-Zehnder interferometer. And the last one considered is a Michelson-Morley interferometer. These geometries will be discussed in the next sections. Before the interferometers are 
discussed, it is explained how a bidirectional coupler works as the fiber-optic equivalent of a beam splitter.

\section{Fiber-Optic Bidirectional Coupler}

Optical fibers are very thin glass fibers which act as waveguides to light. Because of total internal reflection inside the glass fiber, the light is guided through the fiber. A full description of light waveguides and their applications is given by Chen [13]. When two optical waveguides are brought in close proximity of each other, the electromagnetic fields of the light waves start to interact, changing the propagation behavior of the light through the fibers. Depending on the properties of the waveguides, there can be a significant power exchange between them. It is possible to adapt the interaction of two waveguides in such a way that they will act similar to a beam splitter. Therefore, a considerable part of the waveguides have to be parallel and should exhibit the same or almost the same phase velocity. The characteristics of such a bidirectional coupler can be described by using the transfer matrix approach [13]. Let $i_{1}$ and $i_{2}$ be the amplitudes of the light waves at the two inputs of the directional coupler and $o_{1}$ and $o_{2}$ the same for the outputs (see Fig. 3), then for couplers with identical waveguides

$$
\left(\begin{array}{c}
o_{1} \\
o_{2}
\end{array}\right)=\frac{1}{\sqrt{1+\eta^{2}}}\left(\begin{array}{cc}
\eta e^{i \phi_{\eta}} & -i \\
-i & \eta e^{-i \phi_{\eta}}
\end{array}\right)\left(\begin{array}{l}
i_{1} \\
i_{2}
\end{array}\right)
$$

where

$$
\begin{gathered}
\eta=\sqrt{\frac{\sin ^{2} \delta \sin ^{2} \zeta+\cos ^{2} \zeta}{\cos ^{2} \delta \sin ^{2} \zeta}}=\cot \zeta+\frac{\delta^{2}}{\sin 2 \zeta}+O\left(\delta^{4}\right), \\
\tan \phi_{\eta}=\tan \zeta \sin \delta,
\end{gathered}
$$

in which $\delta$ is the mismatch parameter of the coupler, normally close to 0 and the effective length of the coupler is determined by $\zeta=L \sqrt{k_{1} k_{2}\left(1+\delta^{2}\right)}$, where $L$ is the length of the interaction region and $k_{1}$ and $k_{2}$ are the wave vectors of the waves in both waveguides. For an ideal coupler $\delta=0$ and $\zeta=\pi(1+2 p) / 4$, where $p$ is a positive integer. Hence, $\eta=1$ and $\phi_{\eta}=0$. The $2 \times 2$ matrix is referred to as transfer matrix and describes the propagation of the beam through the coupler [13]. For an ideal coupler, when only one of the entrance waveguides is supplied with light, the output of both exit waveguides can be inferred by taking $i_{1}=1$ and $i_{2}=0$. Then, according to Eq. (21),

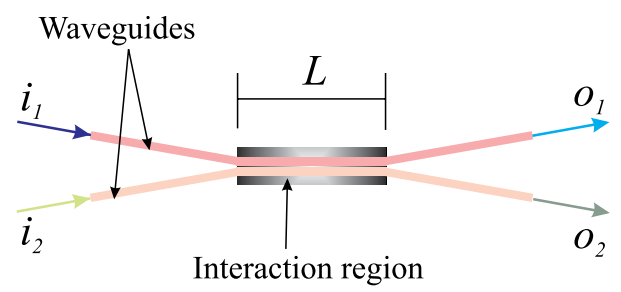

Fig. 3. (Color online) Sketch of fiber-optic bidirectional coupler.

$$
\left(\begin{array}{c}
o_{1} \\
o_{2}
\end{array}\right)=\frac{1}{\sqrt{1+\eta^{2}}}\left(\begin{array}{c}
\eta e^{i \phi_{\eta}} \\
-i
\end{array}\right) \approx \frac{1}{\sqrt{2}}\left(\begin{array}{c}
1 \\
-i
\end{array}\right) .
$$

The wave energy is split over both exit waveguides. The phase difference between the waves of both exit waveguides is $-\pi / 2$. This is similar to the working of a beam splitter. Inversely, if at both entrances waveguides waves are supplied with the same amplitude and a phase difference of $\Delta \phi$, the output of both exit waveguides can be inferred by taking $i_{1}=1 / \sqrt{2}$ and $i_{2}=\exp (i \Delta \phi) / \sqrt{2}$. Then, according to Eq. (21) ,

$$
\left(\begin{array}{l}
o_{1} \\
o_{2}
\end{array}\right)=\frac{1}{2}\left(\begin{array}{c}
1-e^{i \Delta \phi} i \\
e^{i \Delta \phi}-i
\end{array}\right)
$$

so that the amplitudes of $o_{1}$ and $o_{2}$ are given by $\sqrt{1+\sin \Delta \phi} / \sqrt{2}$ and $\sqrt{1-\sin \Delta \phi} / \sqrt{2}$, again similar to the interference effect after the joining of two light beams at a beam splitter.

\section{Sagnac Geometry}

An important type of interferometer is the Sagnac interferometer. It typically splits an incoming light wave into two equal amplitude components which travel through an optical circuit in opposite directions. After traversing the circuit, the waves are joined again and the interfering intensities are detected. This is schematically shown in Fig. 4. Included in the setup is an object with possibly an absorbing layer (representing a standing wave detector). It is inserted approximately half way in the circuit to minimize possible nonreciprocity effects. The collimators are used to couple light out and into the fibers. The fiber stretchers change the optical path of the light waves. These stretchers are optical fibers wrapped around a thin walled cylinder made of piezo material. When applying a voltage over the wall of the cylinder, the piezo material is either contracted or expanded, reducing or extending the length of the fiber wrapped around it. When the fiber stretches, the optical path length changes accordingly and the phase of one light beam with respect to the other is changed.

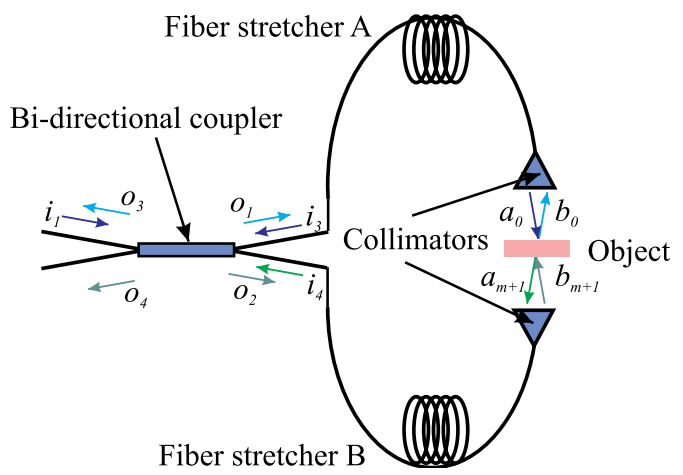

Fig. 4. (Color online) Sketch of fiber-optic Sagnac interferometer. The different directions of the light waves on top and at the bottom of the object are only for display purposes. 
The incident light wave presented at the first entrance waveguide of the bidirectional coupler, $i_{1}$, is provided by a He-Ne laser with a wavelength of $632.8 \mathrm{~nm}$. The other entrance waveguide is not supplied with an incident light beam. Hence, the light wave is split into the two exit waveguides, denoted by $o_{1}$ and $o_{2}$ and given by Eq. (22).

The propagation of the wave through the arms of the interferometer up to the object (including the collimators) can be described by a simple multiplication of the amplitude at the beginning of the arm by the transmission and a phase factor describing the optical path length. Here, it is assumed that the transmission of the fiber is one and the added phase can be described by $\exp \left(i \phi_{A}\right)$ for the arm with fiber stretcher $A$ and $\exp \left(i \phi_{B}\right)$ for the one with fiber stretcher $B$.

Hence, the incident waves at the top and bottom of the object can be calculated from

$$
\left(\begin{array}{c}
a_{0} \\
b_{m+1}
\end{array}\right)=\left(\begin{array}{cc}
e^{i \phi_{A}} & 0 \\
0 & e^{i \phi_{B}}
\end{array}\right)\left(\begin{array}{c}
o_{1} \\
o_{2}
\end{array}\right) .
$$

$b_{0}$ and $a_{m+1}$ can be found by applying the matrix Eq. (10) and rearranging the variables.

$$
\left(\begin{array}{c}
b_{0} \\
a_{m+1}
\end{array}\right)=\left(\begin{array}{cc}
\rho_{t} & \tau \\
\tau & \rho_{b}
\end{array}\right)\left(\begin{array}{c}
a_{0} \\
b_{m+1}
\end{array}\right)
$$

After transmission through or reflection at the object, the bottom and top reflected waves are coupled back into the fiber (note that $\theta_{i}=0$ ) by the collimators and guided back to the bidirectional coupler. The phases accumulated by the waves at the entrance of the bidirectional coupler are

$$
\left(\begin{array}{l}
i_{3} \\
i_{4}
\end{array}\right)=\left(\begin{array}{cc}
e^{i \phi_{A}} & 0 \\
0 & e^{i \phi_{B}}
\end{array}\right)\left(\begin{array}{c}
b_{0} \\
a_{m+1}
\end{array}\right) .
$$

In the bidirectional coupler, the waves interfere and result in the waves at the exit wave guides denoted by $o_{3}$ and $o_{4}$, according to Eq. (21), where $i_{1}$ and $i_{2}$ are replaced by $i_{3}$ and $i_{4}$ and similar replacement for $o_{1}$ and $o_{2}$ :

$$
\left(\begin{array}{c}
o_{3} \\
o_{4}
\end{array}\right)=\frac{1}{\sqrt{1+\eta^{2}}}\left(\begin{array}{cc}
\eta e^{i \phi_{\eta}} & -i \\
-i & \eta e^{-i \phi_{\eta}}
\end{array}\right)\left(\begin{array}{l}
i_{3} \\
i_{4}
\end{array}\right) .
$$

If the Eqs. (22) and (24) $-(\underline{26})$ are inserted, one gets

$$
\left(\begin{array}{c}
o_{3} \\
o_{4}
\end{array}\right)=\frac{e^{i\left(\phi_{A}+\phi_{B}\right)}}{1+\eta^{2}} \times\left(\begin{array}{c}
e^{i \phi_{\eta}}\left(\rho_{t} \eta^{2} e^{i \phi_{s}}-\rho_{b} e^{-i \phi_{s}}-2 i \tau \eta\right) \\
\left(\eta^{2}-1\right) \tau-\eta i\left(\rho_{t} e^{i \phi_{s}}+\rho_{b} e^{-i \phi_{s}}\right)
\end{array}\right)
$$

where $\phi_{s}=\phi_{A}-\phi_{B}+\phi_{\eta}$. Note that $\phi_{s}$ can be changed by either fiber stretcher $A$ or $B$ and that the effect of both stretchers is in opposite directions. Hence, if both increase the optical phase by the same amount, the results do not change. The sum of the intensities at the exit of the bidirectional coupler can be written as

$$
\left|o_{3}\right|^{2}+\left|o_{4}\right|^{2}=T \frac{\left|1+\eta \chi_{t}\right|^{2}+\left|\eta+\chi_{b}\right|^{2}}{1+\eta^{2}}
$$

where

$$
\chi_{t}=\frac{\rho_{t}}{\tau} e^{i\left(\phi_{s}+\pi / 2\right)} \quad \text { and } \quad \chi_{b}=\frac{\rho_{b}}{\tau} e^{-i\left(\phi_{s}+\pi / 2\right)} .
$$

This is a sinusoidal variation on top of a constant level. Note that, if $\rho_{b} \tau^{*}=-\rho_{t}^{*} \tau$ (nonabsorbing object), this reduces to a constant

$$
\left|o_{3}\right|^{2}+\left|o_{4}\right|^{2}=T+R=1 .
$$

For a symmetric object this is

$$
\left|o_{3}\right|^{2}+\left|o_{4}\right|^{2}=T+R-\frac{4 \eta \sqrt{T R} \cos (\arg \rho) \sin \phi_{s}}{1+\eta^{2}} .
$$

Note that for a symmetric and nonabsorbing object $\arg \rho=\pi / 2$.

\section{Mach-Zehnder Geometry}

Another important interferometer is a MachZehnder interferometer. Here, the light wave is split by a beam splitter and the two waves travel along different routes toward a second beam splitter. At the second beam splitter, the light waves are rejoined, resulting in interference between the light waves. In case a standing wave is needed, the interferometer has to be adjusted a bit to provide the standing wave at the object. The way this is done is shown schematically in Fig. 5. Two extra bidirectional couplers above and under the object are added to be able to create a standing wave at the position of the object. The left-hand side of the set-up is similar to the Sagnac configuration with the difference that due to the additional couplers the wave

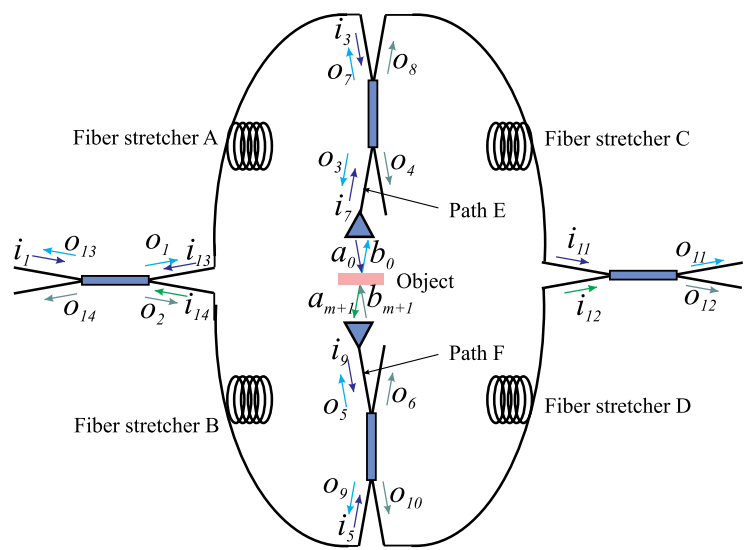

Fig. 5. (Color online) Sketch of fiber-optic Mach-Zehnder interferometer including a standing wave at the object. The different directions of the light waves on top and at the bottom of the object are only for display purposes. 
intensity back after the first coupler is a factor of four less than in the previous section. The right hand side of the set-up couples the same light waves at, but here it is easier to connect a detector to each of the two output ports of the bi-directional coupler.

Again the incident light wave presented at the first entrance wave guide of the most left bi-directional coupler, $i_{1}$ is provided by a He-Ne laser. The other entrance waveguide is not supplied with an incident light beam. The light is split into the two exit waveguides, denoted by $o_{1}$ and $o_{2}$ and given by Eq. (22). The propagation of the wave through the arms of the interferometer up to the next couplers in the path of the light can be described by a simple multiplication of the amplitude at the beginning of the arm by the transmission and a phase factor describing the optical path length. Here, it is assumed that the transmission of the fiber is 1 and the added phase can be described by $\exp \left(i \phi_{A}\right)$ for the arm with fiber stretcher $A$ and $\exp \left(i \phi_{B}\right)$ for the one with fiber stretcher $B$. Hence, the incident waves at the entrance of the next couplers can be calculated from

$$
\begin{aligned}
& \left(\begin{array}{l}
i_{3} \\
i_{4}
\end{array}\right)=\left(\begin{array}{c}
e^{i \phi_{A}} o_{1} \\
0
\end{array}\right), \\
& \left(\begin{array}{l}
i_{5} \\
i_{6}
\end{array}\right)=\left(\begin{array}{c}
e^{i \phi_{B}} o_{2} \\
0
\end{array}\right) .
\end{aligned}
$$

In the bidirectional coupler, the waves interfere and result in the waves at the exit wave guides denoted by $o_{3}, o_{4}$ and $o_{5}, o_{6}$, similar to Eq. (21). Then,

$$
\left(\begin{array}{c}
a_{0} \\
b_{m+1}
\end{array}\right)=\left(\begin{array}{cc}
e^{i \phi_{E}} & 0 \\
0 & e^{i \phi_{F}}
\end{array}\right)\left(\begin{array}{c}
o_{3} \\
o_{5}
\end{array}\right) .
$$

$b_{0}$ and $a_{m+1}$ are calculated from matrix Eq. (25). The waves go further to the couplers where now

$$
\begin{aligned}
\left(\begin{array}{c}
i_{7} \\
i_{8}
\end{array}\right) & =\left(\begin{array}{c}
e^{i \phi_{E}} b_{0} \\
0
\end{array}\right), \\
\left(\begin{array}{c}
i_{9} \\
i_{10}
\end{array}\right) & =\left(\begin{array}{c}
e^{i \phi_{F}} a_{m+1} \\
0
\end{array}\right) .
\end{aligned}
$$

In the encountered bidirectional couplers, the waves interfere again and result in the waves at the exit wave guides denoted by $o_{7}, o_{8}$ and $o_{9}, o_{10}$, similar to Eq. (21). Then outputs $o_{7}$ and $o_{9}$ are transported back to the first coupler, yielding

$$
\left(\begin{array}{l}
i_{13} \\
i_{14}
\end{array}\right)=\left(\begin{array}{cc}
e^{i \phi_{A}} & 0 \\
0 & e^{i \phi_{B}}
\end{array}\right)\left(\begin{array}{l}
o_{7} \\
o_{9}
\end{array}\right) .
$$

In the first coupler, the waves interfere again and result in the waves at the exit wave guides denoted by $o_{13}, o_{14}$ similar to Eq. (21).
Outputs $o_{4}$ and $o_{6}$ can be used to monitor the incident light intensity. With the above equations, the amplitude of the optical wave exiting these wave guides can be shown to be constant:

$$
\left(\begin{array}{c}
o_{4} \\
o_{6}
\end{array}\right)=\frac{-1}{1+\eta^{2}}\left(\begin{array}{c}
-i \eta e^{i\left(\phi_{\eta}+\phi_{A}\right)} \\
e^{i \phi_{B}}
\end{array}\right) .
$$

Further, it can be shown that

$$
\begin{aligned}
\left(\begin{array}{c}
o_{13} \\
o_{14}
\end{array}\right)= & \frac{\eta^{2} e^{i\left(2 \phi_{\eta}+\phi_{A}+\phi_{B}+\phi_{E}+\phi_{F}\right)}}{\left(1+\eta^{2}\right)^{2}} \\
& \times\left(\begin{array}{c}
e^{i \phi_{\eta}}\left(\rho_{t} \eta^{2} e^{i \phi_{s}}-\rho_{b} e^{-i \phi_{s}}-2 i \tau \eta\right) \\
\left(\eta^{2}-1\right) \tau-\eta i\left(\rho_{t} e^{i \phi_{s}}+\rho_{b} e^{-i \phi_{s}}\right)
\end{array}\right),
\end{aligned}
$$

where $\phi_{s}=\phi_{\eta}+\phi_{A}-\phi_{B}+\phi_{E}-\phi_{F}$. This is almost equivalent to Eq. (28). The factor in front of the matrix is different, because now four bidirectional couplers are passed before the light waves reach the output ports and optical paths $E$ and $F$ are added to $A$ and $B$. Outputs $o_{8}$ and $o_{10}$ are transported further to the last coupler, yielding

$$
\left(\begin{array}{l}
i_{11} \\
i_{12}
\end{array}\right)=\left(\begin{array}{cc}
e^{i \phi_{C}} & 0 \\
0 & e^{i \phi_{D}}
\end{array}\right)\left(\begin{array}{c}
o_{8} \\
o_{10}
\end{array}\right) .
$$

In the last coupler, the waves interfere again and result in the waves at the exit wave guides denoted by $o_{11}, o_{12}$ similar to Eq. (21). Using the above equations it can be shown that

$$
\begin{aligned}
\left(\begin{array}{c}
o_{11} \\
o_{12}
\end{array}\right)= & \frac{-i \eta e^{i\left(\phi_{\eta}+\phi_{E}+\phi_{F}+\left(\phi_{A}+\phi_{B}+\phi_{C}+\phi_{D}\right) / 2\right)}}{\left(1+\eta^{2}\right)^{2}} \\
& \times\left(\begin{array}{c}
e^{i \phi_{\eta}}\left(\rho_{t} \eta^{2} e^{i \phi_{s}}-\rho_{b} e^{-i \phi_{s}}-2 i \tau \eta \cos \phi_{m}\right) \\
\left(\eta^{2} e^{i \phi_{m}}-e^{-i \phi_{m}}\right) \tau-\eta i\left(\rho_{t} e^{i \phi_{s}}+\rho_{b} e^{-i \phi_{s}}\right)
\end{array}\right),
\end{aligned}
$$

where $\phi_{s}=\phi_{\eta}+\phi_{E}-\phi_{F}+\left(\phi_{A}-\phi_{B}\right) / 2+\left(\phi_{C}-\phi_{D}\right) / 2$ and $\phi_{m}=\left(\phi_{A}-\phi_{B}\right) / 2-\left(\phi_{C}-\phi_{D}\right) / 2$. Again this is similar to Eqs. (28) and (39). The factor in front of the matrix is different, because now four bidirectional couplers are passed before the light waves reach the output ports and the other waveguides exits of the bidirectional couplers are used. Again, optical paths $E$ and $F$ are added to $A$ and $B$, but also to $C$ and $D$. If $\phi_{C}=\phi_{A}$ and $\phi_{D}=\phi_{B}$, then $\phi_{m}=0$ and the matrix in the above equation reduces to that of Eq. (39).

There are three advantages of this setup over the simple Sagnac geometry. First, both output ports $o_{11}$ and $o_{12}$ can easily be connected to a detector so that the symmetry is not broken. Second, outputs $o_{4}$ and $o_{6}$ can be used to monitor the intensity of the incident wave. And third, the symmetry of the setup is broken so that an extra parameter can be varied and more information obtained about the object.

The sum of the intensities at the exit of the last bidirectional coupler can be written as 


$$
\left|o_{11}\right|^{2}+\left|o_{12}\right|^{2}=\frac{\eta^{2} T}{\left(1+\eta^{2}\right)^{3}}\left(\left|1+\eta \chi_{t}\right|^{2}+\left|\eta+\chi_{b}\right|^{2}\right),
$$

where now

$$
\chi_{t}=\frac{\rho_{t}}{\tau} e^{i\left(\phi_{s}^{\prime}+\pi / 2\right)} \quad \text { and } \quad \chi_{b}=\frac{\rho_{b}}{\tau} e^{-i\left(\phi_{s}^{\prime}+\pi / 2\right)}
$$

and $\phi_{s}^{\prime}=\phi_{s}+\phi_{m}=\phi_{\eta}+\phi_{A}-\phi_{B}+\phi_{E}-\phi_{F}$, independent of $\phi_{C}$ and $\phi_{D}$, as it should because they only distribute the power over the two output waveguides depending of the phase difference between $i_{11}$ and $i_{12} . \phi_{s}^{\prime}$ is the phase difference between the waves traveling toward the object. Again, without absorption this reduces to

$$
\left|o_{11}\right|^{2}+\left|o_{12}\right|^{2}=\frac{\eta^{2}}{\left(1+\eta^{2}\right)^{2}} \approx \frac{1}{4}
$$

because $\eta \approx 1$.

The visibility of this interferometer can be defined as the relative difference between the two outputs of the exit coupler, hence

$$
V=\frac{\left|o_{11}\right|^{2}-\left|o_{12}\right|^{2}}{\left|o_{11}\right|^{2}+\left|o_{12}\right|^{2}}=\frac{\eta^{2}-1}{\eta^{2}+1} V_{1}+V_{2}
$$

Calculating the result from Eq. (41) gives

$$
\begin{aligned}
V_{1} & =\frac{\left|1+\eta \chi_{t}\right|^{2}-\left|\eta+\chi_{b}\right|^{2}}{\left|1+\eta \chi_{t}\right|^{2}+\left|\eta+\chi_{b}\right|^{2}}, \\
V_{2} & =v \cos \left(2 \phi_{m}-\arg \left(1+\eta \chi_{t}\right)+\arg \left(\eta+\chi_{b}\right)\right),
\end{aligned}
$$

where

$$
v=\frac{4 \eta}{\eta^{2}+1} \frac{\left|\left(1+\eta \chi_{t}\right)\left(\eta+\chi_{b}\right)\right|}{\left|1+\eta \chi_{t}\right|^{2}+\left|\eta+\chi_{b}\right|^{2}} .
$$

Note that $V_{1}$ and $v$ are fully determined by the object and the standing wave conditions (determined by $\phi_{s}^{\prime}$ ) on the object. Only $V_{2}$ depends also on the difference between $\phi_{C}$ and $\phi_{D}$ via $2 \phi_{m}$. Hence, by varying $\phi_{m}$ and keeping $\phi_{s}^{\prime}$ constant the visibility changes sinusoidal with an amplitude of $v$. This can be accomplished by moving the object along the optical path. If $\rho_{b} \tau^{*}=-\rho_{t}^{*} \tau$ (nonabsorbing object), this reduces to

$$
\begin{aligned}
V_{1} & =\frac{\left(1-\eta^{2}\right)(2 T-1)+4 \eta T \Re\left\{\chi_{t}\right\}}{1+\eta^{2}}, \\
v & =\frac{4 \eta T}{\left(\eta^{2}+1\right)^{2}}\left|\left(1+\eta \chi_{t}\right)\left(\eta+\chi_{t}\right)\right| .
\end{aligned}
$$

Note that, for bi-directional couplers where $\eta \approx 1$ the first term of Eq. (44) can be neglected.

It is also possible to directly couple the detectors to outputs $o_{8}$ and $o_{10}$, then calculations similar as above result in

$$
\left(\begin{array}{c}
o_{8} \\
o_{10}
\end{array}\right)=\frac{-i \eta e^{i\left(\phi_{\eta}+\phi_{E}+\phi_{F}+\phi_{B}\right)}}{\left(1+\eta^{2}\right)^{(3 / 2)}} \times\left(\begin{array}{c}
\eta \rho_{t} e^{i \phi_{s}^{\prime}}-i \tau \\
e^{i\left(\phi_{F}-\phi_{E}\right)}\left(\eta e^{i \phi_{s}^{\prime}}-\rho_{b} i\right)
\end{array}\right)
$$

where as before $\phi_{s}^{\prime}=\phi_{\eta}+\phi_{A}-\phi_{B}+\phi_{E}-\phi_{F}$. The sum of the intensities is equal to Eq. (42) and the visibility defined similar as before is equal to $V_{1}$.

The advantage of the previous method (i.e. measuring $\left|o_{11}\right|^{2}$ and $\left|o_{12}\right|^{2}$ ) is that the determination of $T$ can be done without an absolute value for the visibility amplitude, as other instrumental factors might influence this. For instance, polarization rotation could reduce the visibility amplitude of the couplers. Let $X$ be the minimum value and $Y$ be the maximum value of the visibility amplitude, then if $\eta \approx 1$,

$$
|2 T-1|=\frac{X}{Y}
$$

The response of the Mach-Zehnder interferometer is similar to the Sagnac interferometer. However, the Sagnac interferometer is much harder to study because of the fact that one input port and output port coincide. Hence, in the following, only the MachZehnder interferometer will be considered.

\section{Michelson-Morley Geometry}

The last important interferometer discussed here is a Michelson-Morley interferometer. The light wave is split by a beam splitter and the two waves travel along different routes toward two separate mirrors. At the mirrors, the light is reflected backwards so that the light waves meet again at the beam splitter. There, the light waves are rejoined, resulting in interference between the light waves at the output ports. The Michelson-Morley geometry is shown schematically in Fig. 6 . The object is inserted in one of the arms between the collimator and the mirror.

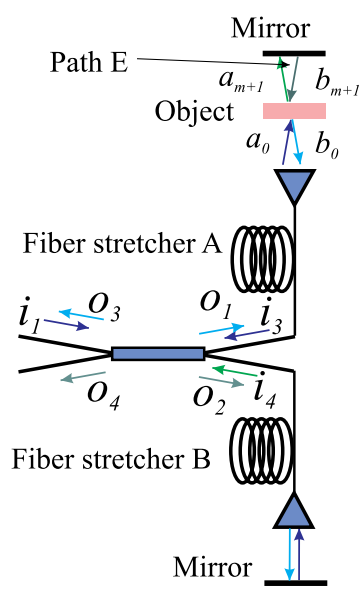

Fig. 6. (Color online) Sketch of fiber-optic Michelson-Morley interferometer including a standing wave at the object. The different directions of the light waves on top and at the bottom of the object are only for display purposes. 
Again, the incident light wave presented at the first entrance wave guide of the most left bidirectional coupler, $i_{1}$, is provided by a He-Ne laser. The other entrance waveguide is not supplied with an incident light beam. The light is split into the two exit waveguides, denoted by $o_{1}$ and $o_{2}$ and given by Eq. (22). The propagation of the wave through the arms of the interferometer up to the next couplers in the path of the light can be described by a simple multiplication of the amplitude at the beginning of the arm by the transmission and a phase factor describing the optical path length. Here, it is assumed that the transmission of the fiber is 1 and the added phase can be described by $\exp \left(i \phi_{A}\right)$ for the arm with fiber stretcher $A$ and $\exp \left(i \phi_{B}\right)$ for the one with fiber stretcher $B$. After the wave passes the object in the upper arm, the light wave is reflected back at the mirror. The optical phase acquired by the light wave between object and mirror is $\phi_{E}$, hence $b_{m+1}=$ $a_{m+1} e^{i\left(2 \phi_{E}+\pi\right)}$ where it was assumed that the reflection coefficient at the mirror is -1 . Applying Eq. (10) yields $b_{0}=-\xi a_{0}$, where

$$
\xi=\frac{m_{12}+m_{22} e^{2 i \phi_{E}}}{m_{11}+m_{21} e^{2 i \phi_{E}}} .
$$

Using the above considerations it can be shown that

$$
\left(\begin{array}{c}
o_{3} \\
o_{4}
\end{array}\right)=\frac{e^{2 i \phi_{B}}}{1+\eta^{2}}\left(\begin{array}{c}
1-\xi \eta^{2} e^{i \phi_{m}} \\
\eta i e^{-i \phi_{\eta}}\left(1+\xi e^{i \phi_{m}}\right)
\end{array}\right)
$$

where $\phi_{m}=2\left(\phi_{\eta}+\phi_{A}-\phi_{B}\right)$. The sum of the intensities at the exit of the bidirectional coupler can be written as

$$
\begin{aligned}
& \left|o_{3}\right|^{2}+\left|o_{4}\right|^{2}=\frac{1+\eta^{2}|\xi|^{2}}{1+\eta^{2}}, \\
& \frac{\left|o_{3}\right|^{2}-\left|o_{4}\right|^{2}}{\left|o_{3}\right|^{2}+\left|o_{4}\right|^{2}}=\frac{\left(1-\eta^{2}\right)\left(1-\eta^{2}|\xi|^{2}\right)-4 \eta^{2} \Re\left\{\xi e^{i \phi_{m}}\right\}}{\left(1+\eta^{2}\right)\left(1+\eta^{2}|\xi|^{2}\right)} .
\end{aligned}
$$

Note that, for a nonabsorbing object $|\xi|=1$, so that

$$
\begin{gathered}
\left|o_{3}\right|^{2}+\left|o_{4}\right|^{2}=1 \\
\frac{\left|o_{3}\right|^{2}-\left|o_{4}\right|^{2}}{\left|o_{3}\right|^{2}+\left|o_{4}\right|^{2}}=\frac{\left(1-\eta^{2}\right)^{2}-4 \eta^{2} \cos \left(\phi_{m}+\arg \xi\right)}{\left(1+\eta^{2}\right)^{2}} .
\end{gathered}
$$

\section{Examples}

\section{A. Nonabsorbing Single Layer}

The most simple object is a single layer with thickness $t$ and refractive index $n$. As this object is symmetric, the top and bottom reflection amplitudes are equal to

$$
\rho=\frac{e^{2 i n q_{0} t}-1}{1-\rho_{F}^{2} e^{2 i n q_{0} t}} \rho_{F},
$$

where the Fresnel reflection amplitude is given by

$$
\rho_{F}=\frac{n-1}{n+1} .
$$

The transmission amplitude is

$$
\tau=\frac{e^{i n q_{0} t}}{1-\rho_{F}^{2} e^{2 i n q_{0} t}} \tau_{F}
$$

where the Fresnel transmission amplitude is

$$
\tau_{F}=\frac{4 n}{(n+1)^{2}} .
$$

Hence, the ratio between $\rho$ and $\tau$ is simply

$$
\frac{\rho}{\tau}=i \sin \left(n q_{0} t\right) \frac{\rho_{F}}{\tau_{F}} .
$$

For a single nonabsorbing layer with a refractive in$\operatorname{dex} n=1.45$ and a thickness corresponding to an optical path of half a wavelength, the visibility of the Mach-Zehnder and Michelson-Morley configurations as function of $\phi_{s}^{\prime}, \phi_{m}$, and $\phi_{E}, \phi_{m}$, respectively, are shown in the bottom part of Fig. 7. In the upper part, the same is shown for a layer thickness corresponding to a quarter wavelength. For a thickness with an optical path of half a wavelength $n q_{0} t=\pi$, hence $\rho=0$. Because there is no reflection at all, the visibility of the interferometers does not depend
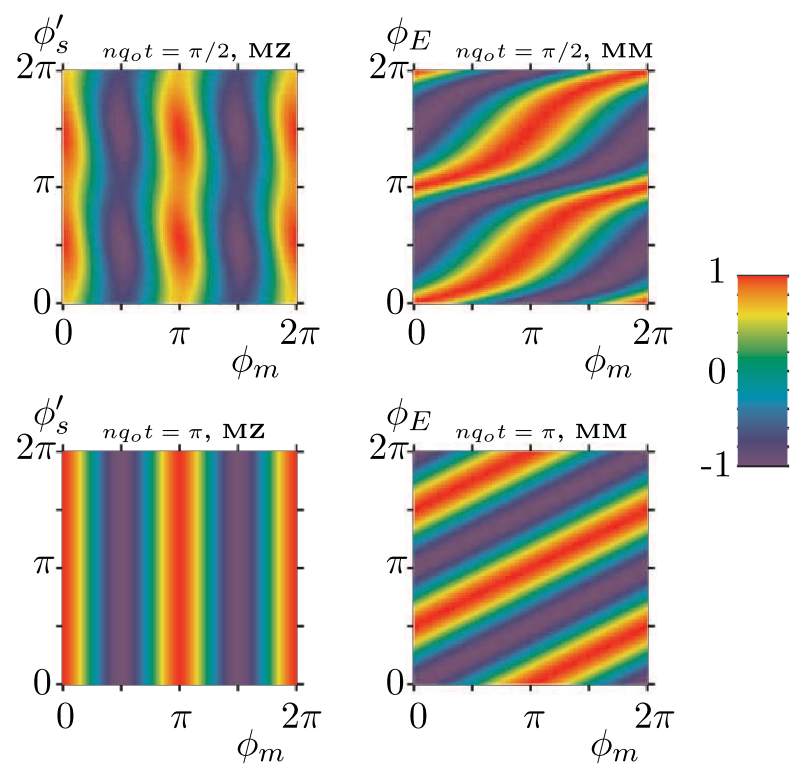

Fig. 7. (Color online) Visibilities of Mach-Zehnder (left) and Michelson-Morley (right) interferometers including a single layer (refractive index $n=1.45$ ) with a thickness corresponding to an optical phase of $\pi / 2$ (top) and $\pi$ (bottom) as function of the two optical phases $\phi_{s}^{\prime}, \phi_{m}$ and $\phi_{E}, \phi_{m}$, respectively. 
on the phase difference between the traveling waves hitting the object (i.e. $\phi_{s}^{\prime}$ and $\phi_{m}-2 \phi_{E}$ ). The visibility response is similar to the one of a standard interferometer. For a thickness with an optical path of a quarter (or three quarter) wavelength $n q_{0} t=\pi / 2$, hence $\rho=-2 \rho_{F} /\left(1-\rho_{F}^{2}\right)$ and is maximal. Now there is reflection and the visibility of the interferometers does depend on the phase difference between the traveling waves hitting the object. Both the visibility responses for the Mach-Zehnder and MichelsonMorley interferometer are changed. Here, for constant $\phi_{s}^{\prime}, \phi_{E}$, the visibility variations are still sinusoidal, although the amplitude of the visibility oscillations decreases. This makes clear why it is important to minimize reflections in interferometers.

\section{B. Strong-absorbing Single Layer}

The most simple absorbing object is a single layer with thickness $t$ and refractive index $n=n_{r}+i \kappa$. The reflection and transmission amplitudes are equal to the ones in the previous section, except now the refractive index is complex. For a silver (at $632.8 \mathrm{~nm}$ the refractive index is $n_{r}=0.135$ and $\kappa=3.99$ ) layer of $15 \mathrm{~nm}$ thickness, the visibility of the MachZehnder and Michelson-Morley configurations as function of $\phi_{s}^{\prime}, \phi_{m}$ and $\phi_{E}, \phi_{m}$, respectively, are shown in the bottom part of Fig. 8 . In the upper part, the same is shown for a layer of titanium (at $632.8 \mathrm{~nm}$ the refractive index is $n_{r}=2.153$ and $\kappa=2.92$ ) with the same thickness. The four graphs are all completely different, stressing the different behavior of the visibility of the interferometers. Note that, for the Michelson-Morley interferometer, the visibility change dependence of $\phi_{E}$ is reduced. Even if $\phi_{E}$ would change its value very fast, the average visibility change of the interferometer by changing $\phi_{m}$ is
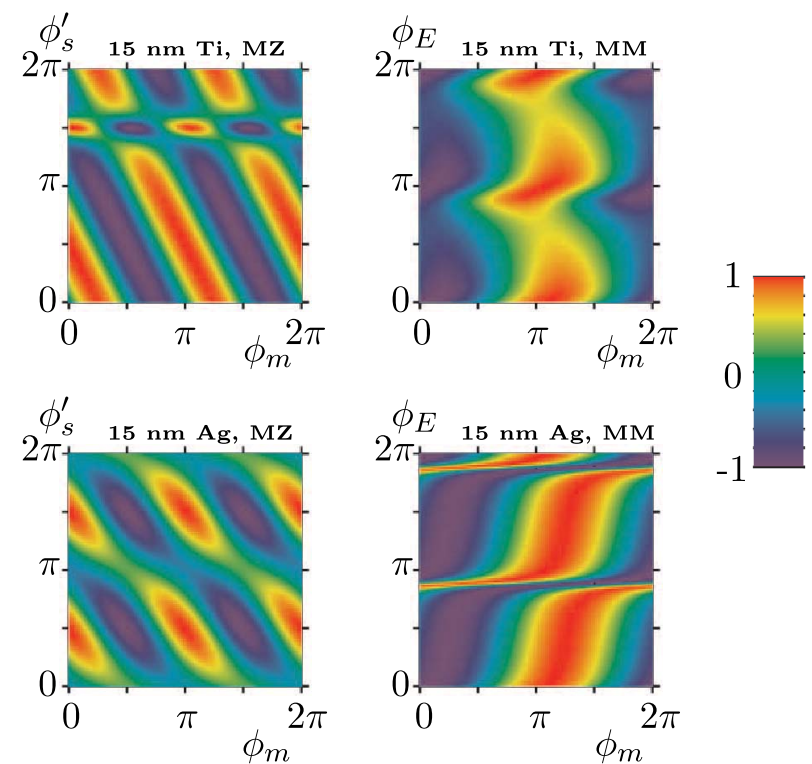

Fig. 8. (Color online) Visibilities of Mach-Zehnder (left) and Michelson-Morley (right) interferometers including a single titanium (top) and silver (bottom) layer of $15 \mathrm{~nm}$ thickness as function of the two optical phases $\phi_{s}^{\prime}, \phi_{m}$ and $\phi_{E}, \phi_{m}$, respectively.
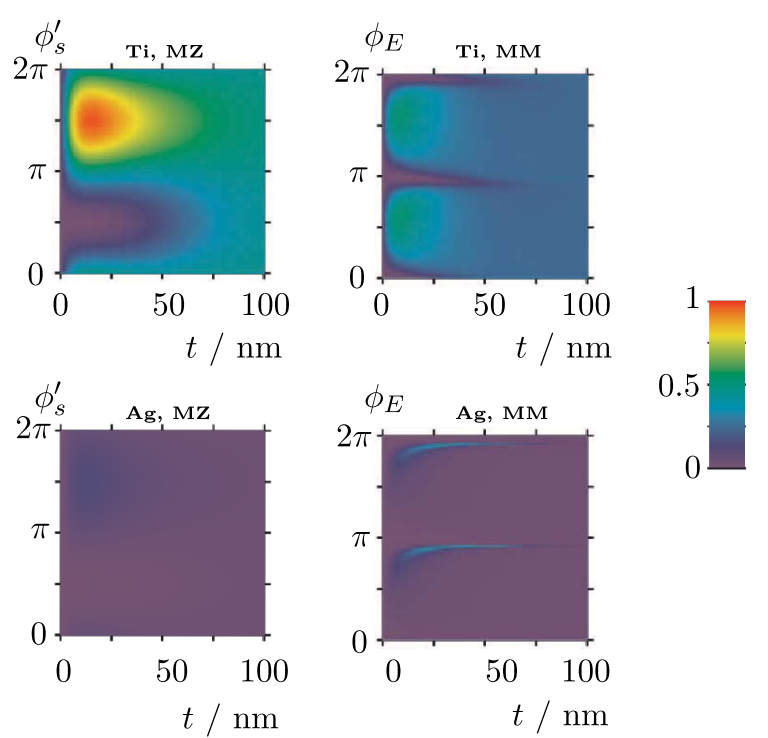

Fig. 9. (Color online) Absorbed fraction in a Mach-Zehnder (left) and Michelson-Morley (right) interferometers including a single titanium (top) and silver (bottom) layer of varying thicknesses as function of the optical phase between the propagating waves, $\phi_{s}^{\prime}$ and $\phi_{E}$, respectively.

not reduced to 0 . Hence, the interferometer looses its sensitivity for $\phi_{E}$. Off course, this is logical if the object would be a perfect reflector.

In Fig. 9 , it is shown that the absorption for these objects is also different in the two interferometers and depends in a nonregular way on the thickness of the layer.

\section{Weak-absorbing Single Layer}

If the absorption is less, then the absorbed fraction starts to oscillate with the thickness of the layer. This is shown for a layer with a real part of the refractive index of 1.8 and an imaginary part of 0.02 in Fig. 10.

\section{Fabry-Pérot Cavity}

With two semitransparent mirrors a Fabry-Pérot cavity can be constructed. Here, the semitransparent mirrors are made out of a thin single layer of metal on a piece of glass as schematically shown in Fig. 11. The mirrors can be positioned in four different ways to form the cavity: (a) glass substrates facing each
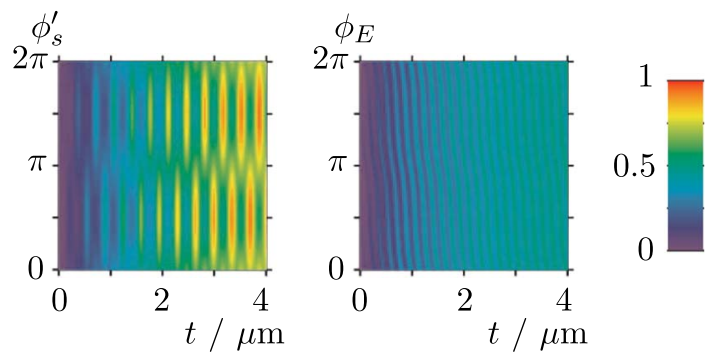

Fig. 10. (Color online) Absorbed fraction in a Mach-Zehnder (left) and Michelson-Morley (right) interferometers including a single layer of varying thicknesses as function of the optical phase between the propagating waves, $\phi_{s}^{\prime}$ and $\phi_{E}$, respectively. The refractive index of the layer is $n=1.8+0.02 i$. 


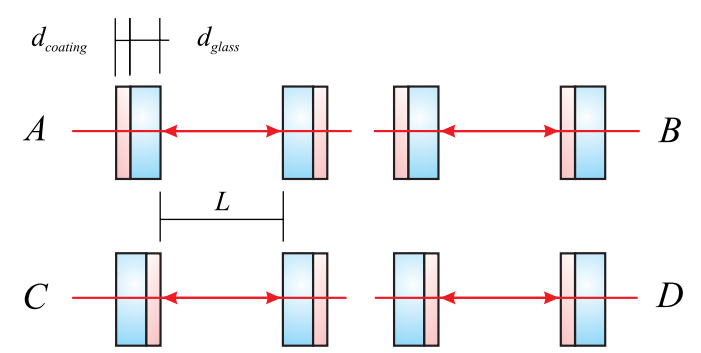

Fig. 11. (Color online) Schematic of Fabry-Pérot cavity with length, $L$. Each semitransparent mirror consists of a thin metal layer on top of a glass substrate. The mirrors can be positioned in four different ways to form the cavity: (a) glass substrates facing each other, (b) and (c) glass substrate facing metal layer, and (d) metal layers facing each other.

other, (b) and (c) glass substrate facing metal layer, and (d) metal layers facing each other. The length of the cavity can be varied. The result for a MachZehnder interferometer for a metal layer of $15 \mathrm{~nm}$ titanium on top of a $3 \mathrm{~mm}$ thick glass $(n=1.45)$ substrate is shown in Fig. 12. Again, the absorbed fraction depends on the phase difference between the traveling wave and also on the length of the cavity and how the cavity is constructed. If the metal layers are facing each other $(D)$, the waves inside the cavity at reflection first encounter the absorbing metal layer increasing the absorption with respect to the situation when the glass substrates are facing each other $(A)$. For a suitable phase difference between the traveling waves and an appropriate cavity length, the absorption virtually vanishes. If the metal layer of one mirror faces the glass substrate of the other $(B$ and $C$ ), the absorption is in between these extremes. The result for the same type of semitransparent
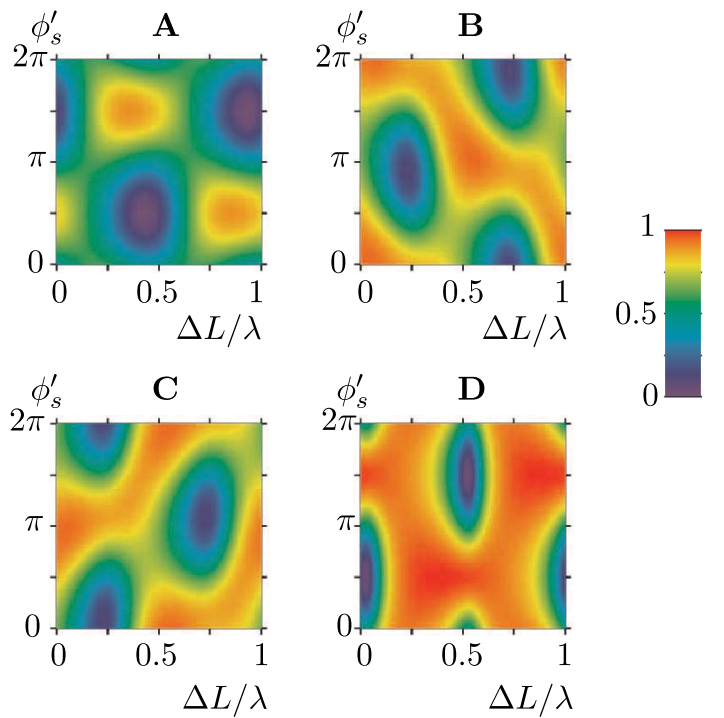

Fig. 12. (Color online) Absorbed fraction in a Mach-Zehnder interferometer as function of the optical phase between the propagating waves, $\phi_{s}^{\prime}$ and the varying length of a Fabry-Pérot cavity for geometries (a) to (d) (see Fig. 11). The semitransparent mirrors consist of a single layer of $15 \mathrm{~nm}$ titanium of top of a $3 \mathrm{~mm}$ glass substrate.
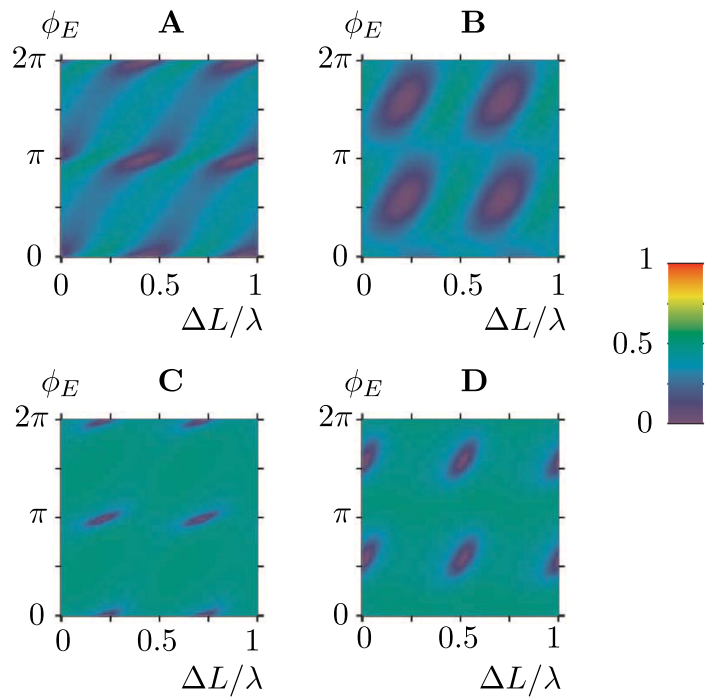

Fig. 13. (Color online) Absorbed fraction in a Michelson-Morley interferometer as function of the optical phase between the propagating waves, $\phi_{E}$ and the varying length of a Fabry-Pérot cavity for geometries (a) to (d) (see Fig. 11). The semitransparent mirrors consist of a single layer of $15 \mathrm{~nm}$ titanium of top of a $3 \mathrm{~mm}$ glass substrate.

mirror and a Michelson-Morley interferometer is shown in Fig. 13. If the metal layers are facing each other $(D)$, the waves inside the cavity at reflection first encounter the absorbing metal layer increasing the absorption with respect to the situation when the glass substrates are facing each other $(A)$. For a suitable phase difference between the traveling waves and an appropriate cavity length, the absorption virtually vanishes. If the metal layer of one mirror faces the glass substrate of the other $(B$ and $C$ ), the absorption pattern is almost inverted. The more complex behavior of the absorption in this interferometer is due to the a-symmetry of the Michelson-Morley setup. A second cavity is created between the mirror of the interferometer and the second mirror of the cavity. The result for a Mach-Zehnder interferometer for a metal layer of $15 \mathrm{~nm}$ silver on top of a $3 \mathrm{~mm}$ thick glass $(n=1.45)$ substrate is shown in Fig. 14. Here the absorption is much less, due to the small real part of the refractive index. The patterns are more symmetric, but similar differences as in the previous case can still be recognized. The result for the same type of semitransparent mirror and a Michelson-Morley interferometer is shown in Fig. 15.

From these examples, it is clear that, as soon as standing waves are considered in interferometers, the absorption phenomena in the objects placed in the interferometer depend strongly on the properties of the created standing waves.

\section{Experiments}

To check if the above described effects are observable in fiber-optic interferometers, measurements were performed with a Mach-Zehnder interferometer corresponding to Fig. 5 and a Michelson-Morley interferometer corresponding to Fig. 6 . The object 

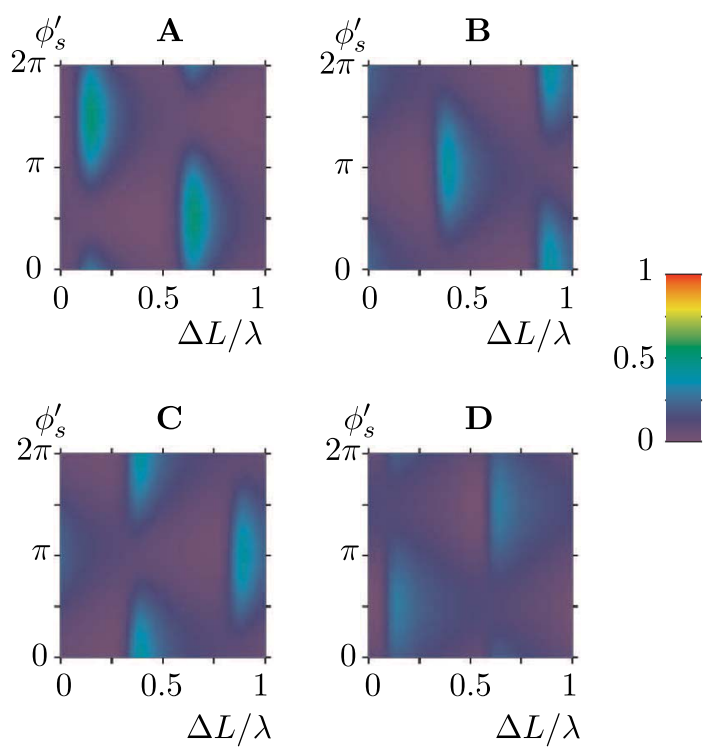

Fig. 14. (Color online) Absorbed fraction in a Mach-Zehnder interferometer as function of the optical phase between the propagating waves, $\phi_{s}^{\prime}$ and the varying length of a Fabry-Pérot cavity for geometries (a) to (d) (see Fig. 11). The semitransparent mirrors consist of a single layer of $15 \mathrm{~nm}$ silver of top of a $3 \mathrm{~mm}$ glass substrate.

consisted of a glass (BK7) substrate (thickness $3 \mathrm{~mm}$ ) with a layer of silver (thickness $14.7 \mathrm{~nm}$ ) deposited on it at one side. The light of a stabilized He-Ne laser (type Coherent 200 , linear polarized, $0.5 \mathrm{~mW}$, maximum mode sweep $10 \mathrm{MHz}$ ) was coupled into the $i_{1}$ arms of the interferometers via a polarization dependent optical isolator (isolation at least $35 \mathrm{~dB}$ ). The interferometers were put in a temperature controlled environment where the temperature control was
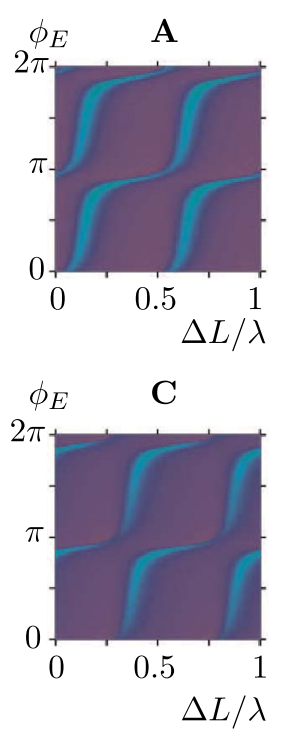

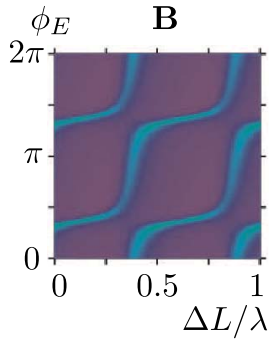

$\phi_{E} \quad \mathrm{D}$

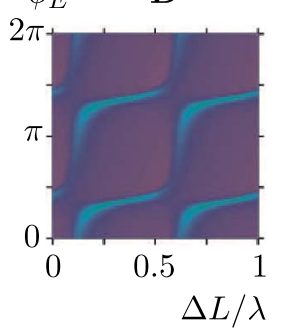

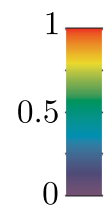

0
Fig. 15. (Color online) Absorbed fraction in a Michelson-Morley interferometer as function of the optical phase between the propagating waves, $\phi_{E}$ and the varying length of a Fabry-Pérot cavity for geometries (a) to (d) (see Fig. 11). The semitransparent mirrors consist of a single layer of $15 \mathrm{~nm}$ silver of top of a $3 \mathrm{~mm}$ glass substrate.
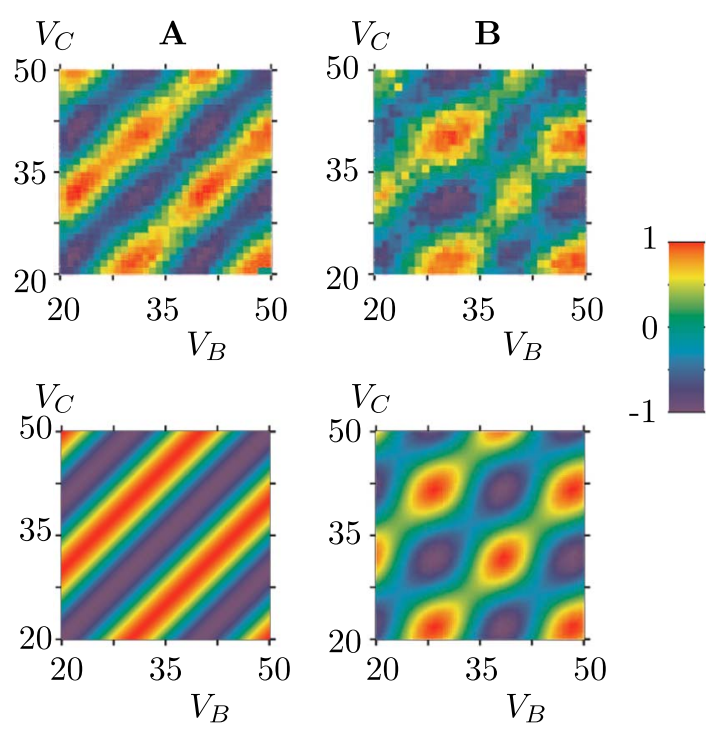

Fig. 16. (Color online) Measured (top) and theoretical (bottom) renormalized visibility of a Mach-Zehnder interferometer as function of the voltage applied to fiber stretchers $B$ and $C$. (a) without object; (b) object consisting of a single layer of $14.7 \mathrm{~nm}$ silver on top of a $3 \mathrm{~mm}$ glass substrate.

within $3 \mathrm{mK}$. For the Mach-Zehnder geometry the fiber stretchers were made from $1 \mathrm{~m}$ long SM600 fibers wrapped around a thin-walled cylinder made of piezo material. In the Michelson-Morley geometry, the fiber stretchers were omitted, but the mirrors were mounted by means of piezo stacks enabling the independent change of the optical paths. The light intensity at outputs $o_{11}$ and $o_{12}$ of the Mach-Zehnder interferometer was measured by two amplified silicon detectors. The visibilities can be calculated from these intensities according to Eq. (44). After normalizing the maximum visibility to 1 , they are shown in Fig. 16 as function of the voltage applied to the fiber stretchers $B$ and $C$ for the measurements without the object (a) and with the object (b). The theoretical values are sensitive to the exact glass thickness $(3000025 \mathrm{~nm})$ and refractive index (1.515). The sum of these intensities relative to the output intensity of the laser for the same data is given in Figs. 17(a) and 17(b). The modulation visible in Figs. 16(a) and 17(a) indicates that already the interferometer without an object contains absorbing parts or reflecting interfaces. This probably is due to the used fibers and applied antireflecting coatings. From Fig. 17, it is clear that the insertion of the object in the optical path reduces the light intensity at the output of the interferometer. Part of this reduction is due to the absorption in the object, part is due to reflection at the object. From these figures it is evident that by changing the optical path corresponding to fiber stretcher $C$, the sum of the intensities does not change. This is due to the fact that the standing wave at the position of the object does not change. The relative change in absorption as function of $V_{B}$ is increased, showing the influence of the phase of the standing wave on the absorption of light in the object. 

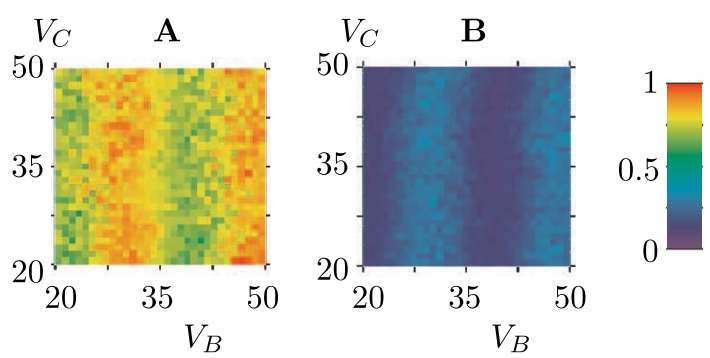

Fig. 17. (Color online) Measured renormalized sum of intensities of outputs of a Mach-Zehnder interferometer as function of the voltage applied to fiber stretchers $B$ and $C$. (a) without object; (b) object consisting of a single layer of $14.7 \mathrm{~nm}$ silver on top of a $3 \mathrm{~mm}$ glass substrate.

The light intensity at output $o_{4}$ of the MichelsonMorley interferometer was also measured as function of the voltage applied to the mirror piezo stacks 1 and 2 . The results normalized to the laser intensity is shown in Fig. 18 for the measurements without the object (a) and with the object (b). The theoretical calculations have been done for the same parameters as above. A slight modulation is visible in Fig. 18(a). The slight curvature of the intensity should not exist if an object is not present. However, as has been shown in Figs. 7 (right), a simple glass substrate as an object could already cause these effects. As the interferometer is made out of optical fibers, there are quite a few air-glass and glass-air interfaces. Their combined effect can easily explain the observed features.

From Figs. 16(b) and 18(b), it is clear that the insertion of the object in the optical path changes the behavior of the output of the interferometers as function of the phase of the standing wave crossing the object. As can be inferred from Figs. $\underline{8}$ and $\underline{9}$,
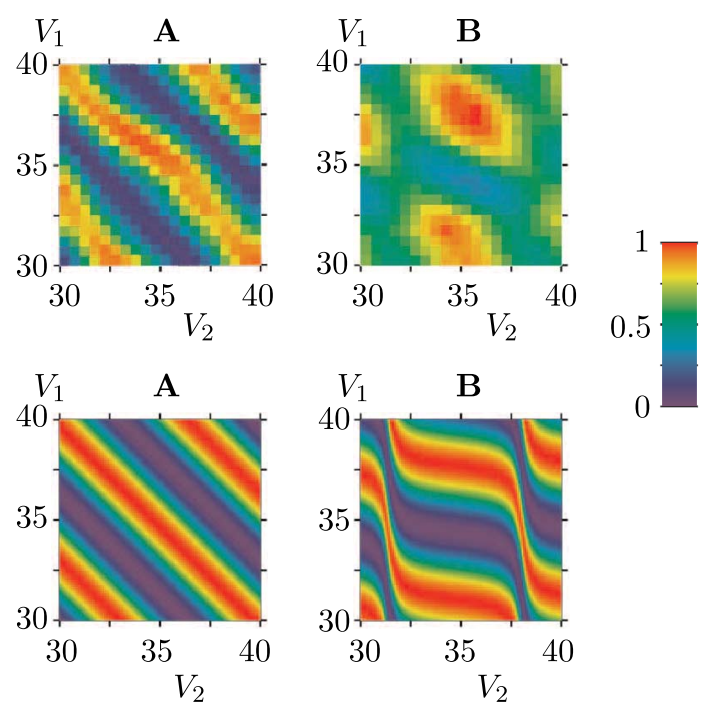

Fig. 18. (Color online) Measured (top) and theoretical (bottom) renormalized intensity of output of a Michelson-Morley interferometer as function of the voltages applied to mirror piezo stacks. (a) without object; (b) object consisting of a single layer of $14.7 \mathrm{~nm}$ silver on top of a $3 \mathrm{~mm}$ glass substrate. the response of the interferometers is very much dependent on the exact properties of the absorbing layer. The theoretical calculations of Fig. 16(b) and 18(b) were performed with literature values for the refractive index and an independently measured value of the thickness of the silver layer. Better correspondence could be achieved by fitting these values. However, this would not add to the insight and fitted plots are omitted here.

In this case, the deviation between the theoretical calculations and the measurements is larger for the Michelson-Morley geometry than with the MachZehnder geometry. This is due to the different way in which the standing wave is created in the interferometers. In the former case the standing wave is created directly by reflection to a mirror. In the latter, it is created by splitting it at the bidirectional coupler. The reduction of the deviations between the two is left to the interested reader.

\section{Conclusions}

The different behavior of the standing waves in the interferometers is caused by the different ways the standing waves are produced and the different ways the object reacts on them. As long as the object is nonabsorbing and the reflectivity is kept low, the influence on the interferometers is small. As soon as considerable absorption or reflection occurs, the visibility changes of the interferometers become more complex. For the Mach-Zehnder interferometer, the visibility changes sinusoidal as long as the phase difference of the incident waves on the object remains the same. For the Michelson-Morley interferometer, the visibility changes are more complex. Experiments in a temperature controlled environment confirm the complex behavior of the visibility changes and are compatible with the presented calculations. Although the measurements show similar characteristics compared to the theoretical calculations, further experiments are needed for a thorough comparison between theory and experiment.

\section{References}

1. F. A. Jenkins and H. E. White, Fundamentals of Optics (McGraw-Hill, 1981).

2. E. Hecht and A. Zajac, Optics (Addison-Wesley, 1979).

3. L. Mandel and E. Wolf, Optical Coherence and Quantum Optics (Cambridge University, 1995).

4. J. E. M. Lekner, Theory of Reflection (Martinus Nijhoff, 1987).

5. O. Wiener, "Stehende Lichtwellen und die Schwingungsrichtung polarisirten Lichtes," Ann. Phys. (Berlin) 276, 203 (1890).

6. H. E. Ives and T. C. Fry, "Standing light waves; repetition of an experiment by Wiener, using a photoelectric probe surface," J. Opt. Soc. Am. 23, 73-83 (1933).

7. E. W. Silvertooth and S. F. Jacobs, "Standing wave sensor," Appl. Opt. 22, 1274-1275 (1983).

8. L. Carraresi, E. A. De Souza, D. A. B. Miller, W. Y. Jan, and J. E. Cunningham, "Wavelength-selective detector based on a quantum well in a standing wave," Appl. Phys. Lett. 64, 134136 (1994) 
9. M. Sasaki, X. Mi, and K. Hane, "Standing wave detection and interferometer application using a photodiode thinner than optical wavelength," Appl. Phys. Lett. 75, 2008 (1999).

10. H.-J. Büchner, H. Stiebig, V. Mandryka, E. Bunte, and G. Jäger, "An optical standing-wave interferometer for displacement measurements," Meas. Sci. Technol. 14, 311 (2003).
11. Y. Li, X. Mi, M. Sasaki, and K. Hane, "Precision optical displacement sensor based on ultra-thin film photodiode type optical interferometers," Meas. Sci. Technol. 14, 479 (2003).

12. L. D. Landau and E. M. Lifshitz, Electrodynamics of Continuous Media in Vol. 8 of Course of Theoretical Physics, 2nd ed. (Elsevier, 1984).

13. C. L. Chen, Foundations for Guided-Wave Optics (Wiley, 2007). 